\title{
Emergent quantum matter in graphene nanoribbons
}

\author{
J. L. Lado, ${ }^{1,2}$ R. Ortiz, ${ }^{3}$ and J. Fernández-Rossier ${ }^{4}$ \\ ${ }^{1}$ Institute for Theoretical Physics, ETH Zurich, 8093 Zurich, Switzerland \\ ${ }^{2}$ Department of Applied Physics, Aalto University, Espoo, Finland \\ ${ }^{3}$ Departamento de Física Aplicada, Universidad de Alicante, 03690 Spain \\ ${ }^{4}$ QuantaLab, International Iberian Nanotechnology Laboratory, \\ Avenida Mestre José Veiga, 4715-330 Braga, Portugal
}

\begin{abstract}
In this book chapter, we introduce different schemes to create quantum states of matter in engineered graphene nanoribbons. We will focus on the emergence of controllable magnetic interactions, topological quantum magnets, and the interplay of magnetism and superconductivity. We comment on the experimental signatures of those states stemming from their electronic and spin excitations, that can be observed with atomic resolution using scanning probe techniques.
\end{abstract}

\section{INTRODUCTION}

In this chapter we provide a perspective on the potential of graphene nanoribbons as a platform to host a variety of non-trivial emergent electronic states, such as topological phases, quantum spin liquids, broken symmetry magnetic states and Yu-ShibaRusinov excitations. This potential arises from the capability to nanoengineer the electronic properties of nanoribbons using several different resources:

- Geometrical control. Graphene ribbons with different shapes, orientation, widths, can be synthesized[1]. This gives rise to different electronic properties, including the emergence of localized edge and interface states that can host unpaired spin electrons

- Tuning the electron density. Using either gating and chemical doping it is possible to control the density of electrons.

- The electronic properties of GNR can be affected by several types of proximity effect: spin-orbit, superconducting and magnetic. Therefore, they provide an unique platform to explore the interplay between local magnetic order and superconductivity.

In addition, scanning probe spectroscopies permit to probe both the structural properties of GNR as well as their electronic properties [2, 6] and spin excitations [7], with atomic scale resolution, and constitute great tool to probe the emergent electronic phases. [8]

The most compelling argument to expect nontrivial correlated phases in GNR comes from experiments. Non-trivial phases, including MottHubbard insulating phases[9] and non-trivial superconductivity[10] have been observed in twisted graphene bilayers. Whereas the precise origin of the superconductivity is not understood, there is a consensus on the crucial role played by an array of localized states that form very narrow bands at the Dirac energy. Given that the chemical properties of monolayer GNR are almost identical to those of graphene bilayer, it is our contention that the localized zero modes of the GNR can also result in non-trivial correlated phases. The scope of the chapter is to unveil some physical mechanisms that can promote non-trivial electronic phases, rather than the technical aspects of how to model them.

We focus on three classes of non-trivial electronic behavior. First, we revisit the thoroughly studied problem of magnetic order in the zigzag edges. We address the prominent role of spin fluctuations in this low dimensional system. Second, we discuss how to realize spin chain Hamiltonians in graphene ribbons engineered to host an ordered array of localized zero mode states. Third, we address the interplay between emergent local moments and superconducting proximity effect.

\section{MODELING GNR}

\section{Geometries}

In this chapter we focus on GNR with atomically precise edges along the crystallographic axis of graphene. These are the so called armchair and zigzag edges. We consider both finite (OD) and infinite (1D) ribbons. We also consider ribbons with a periodic modulation of their width, that are known to host interfacial topological zero modes [11-14]. Several other geometries, such as chiral ribbons with sufficiently long zigzag patches, as well as chevron type ribbons with zigzag edges, can result in the formation of local moments and non-trivial spin physics. 

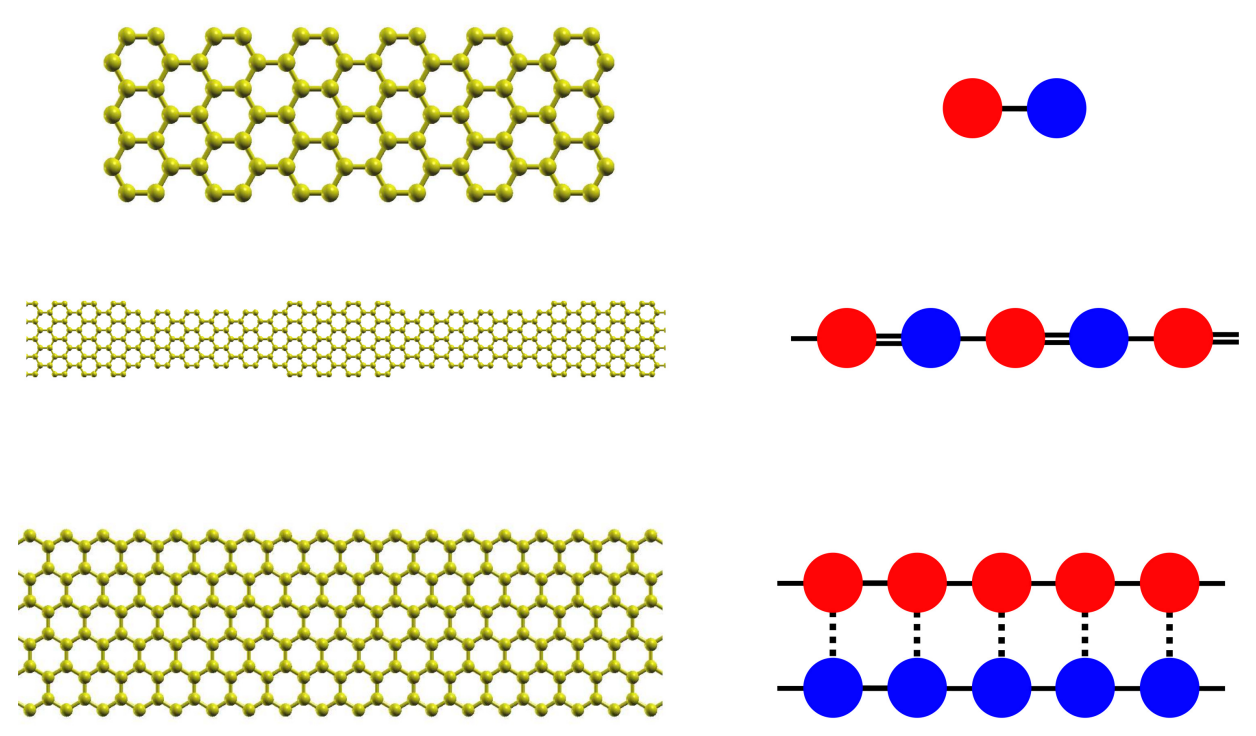

FIG. 1. Left column different GNR considered in this chapter. Top: a finite size graphene ribbon. Middle: 1D graphene ribbon that alternates to sections with different width and armchair edge. Bottom: a 1D ribbon with zigzag edges. Right column: the equivalent lattice spin model.

\section{Single particle terms}

Here we adopt the standard model [15, 16] to describe GNR, namely, a single orbital tight-binding model with first neighbor hopping $t$. The single orbital is the $p_{z}$ atomic orbital of carbon, that is decoupled from the rest in planar structures. Unless otherwise stated, we assume that edge carbon atoms are passivated with hydrogen, so that there are no dangling bonds at the surface. This model gives a fair description of the states in a few $\mathrm{eV}$ window around the Fermi energy for planar carbon based structures, going from zero dimensional molecules, to planar graphene. It is standard[15] to take $t=-2.7 \mathrm{eV}$, which provides a good slope for the Dirac cones in planar graphene. The first neighbor hopping Hamiltonian reads:

$$
\mathscr{H}_{0}=-t \sum_{i, i^{\prime}, \sigma} c_{i \sigma}^{\dagger} c_{i^{\prime}, \sigma}
$$

where $i^{\prime}$ stand for the first neighbors of $i$. This is the dominant term in the Hamiltonian and it accounts for the Dirac cones in graphene [15, 16], the existence of localized states in the zigzag edges[17], and the gapped bands in armchair GNR[17].

In addition, we consider the effect of the several spin-dependent terms in the Hamiltonian. First, the Zeeman term, given by

$$
\mathscr{H}_{Z}=\frac{1}{2} g \mu_{B} \vec{B} \cdot \sum_{i, \sigma, \sigma^{\prime}} \vec{\sigma}_{\sigma, \sigma^{\prime}} c_{i \sigma}^{\dagger} c_{i, \sigma^{\prime}}
$$

where $g \simeq 2$ and $\vec{\sigma}_{\sigma, \sigma^{\prime}}$ are the Pauli matrices.

The intrinsic spin-orbit coupling, proposed by Kane and Mele, is described by [18]:

$$
H_{K M}=\sum_{i, i^{\prime \prime} \sigma} i t_{K M} \sigma v_{i, i^{\prime \prime}} c_{i \sigma}^{\dagger} c_{i^{\prime \prime} \sigma}
$$

where $i^{\prime \prime}$ stands for the second neighbors of $i$, summation, $\sigma= \pm 1$ are the spin projections (along the axis perpendicular to the crystal plane) and $v_{i, i^{\prime \prime}}=+(-) 1$ for clockwise (anticlockwise) second neighbor hopping. When added to the hopping Hamiltonian (1), the Kane-Mele term opens a topologically non-trivial band-gap $\Delta_{S O C}=6 \sqrt{3} t_{K M}$ at the Dirac points. The non-trivial nature of the gap implies the emergence of spin-locked chiral edge states[18]. Because of the small magnitude of $\Delta_{S O C}$ in graphene, the observation of this gap is very challenging[19] and the localization length of the edge states is very large. Therefore, this term has a minor influence in the properties of graphene ribbons. However, this type of term could be enhanced by proximity effect[20].

A second type of spin obit effect can arise when mirror symmetry is broken, due to the application of an external off-plane electric field, or due to interaction with the substrate. This is the so called Rashba spin-orbit term [18, 21]:

$$
\mathscr{H}_{R}=i t_{R} \sum_{i, j, s, s^{\prime}} \vec{E} \cdot\left(\vec{r}_{i j} \times \vec{\sigma}\right)_{s, s^{\prime}} c_{i s}^{\dagger} c_{j s^{\prime}}
$$

where $\vec{r}_{i j}$ is unit vector along the bond between the carbon sites $i$ and $j, \vec{\sigma}$ are the spin Pauli matrices and 
$\vec{E}$ is a vector related to inversion symmetry breaking of the graphene lattice, such as an off-plane electric field[13, 21]. The Rashba spin orbit coupling does not commute with $S_{z}$ and promotes mixing between the two spin channels.

\section{Coulomb interaction}

In this chapter we consider the effect of electronelectron Coulomb interactions within the Hubbard approximation:

$$
\mathscr{H}_{U}=U \sum_{i} n_{i \uparrow} n_{i \downarrow}
$$

where $U$ stands for the Coulomb penalty for having 2 electrons in the same $\pi$ orbital in a single carbon atom. The value of $U$ may depend on additional screening effects, including the substrate. In addition, the right value of $U$ might depend on whether or not we include a next-neighbor Coulomb repulsion in the Hamiltonian[22]. Here we adopt $U$ as a variable parameter, that takes values in the range of $U=|t|$.

The Hubbard model can only be solved exactly in very specific geometries, such as the monostrand one dimensional chain. Thus, very often[23-29] the model is treated at the mean field approximation, where the exact Hamiltonian is replaced by an effective Hamiltonian

$$
\mathscr{H}_{U, M F}=\mathscr{H}_{\text {Hartree }}+\mathscr{H}_{\text {Fock }}
$$

where

$$
\begin{gathered}
\mathscr{H}_{\text {Hartree }}=U\left(n_{i, \uparrow}\left\langle n_{i, \downarrow}\right\rangle+n_{i, \downarrow}\left\langle n_{i, \uparrow}\right\rangle\right) \\
\mathscr{H}_{\text {Fock }}=-U\left(c_{i, \downarrow}^{\dagger} c_{i, \uparrow}\left\langle c_{i, \uparrow}^{\dagger} c_{i, \downarrow}\right\rangle+c_{i, \uparrow}^{\dagger} c_{i, \downarrow}\left\langle c_{i, \downarrow}^{\dagger} c_{i, \uparrow}\right\rangle\right)
\end{gathered}
$$

so that electrons interact with an external field that is self-consistently calculated. Most often [23-26, 3032] an additional approximation has been used, that assumes a collinear magnetization so that the Fock term vanishes. For small nanographenes, such as triangular and hexagonal islands with zigzag edges [24], the results of collinear mean-field calculations of the Hubbard model are very similar to those obtained using density functional theory calculations that include long-range Coulomb interactions and include several atomic orbitals per carbon atom. The same statement holds true for infinitely long graphene ribbons with zigzag edges: both mean field calculations Hubbard model calculations [23, 25] and DFT based calculations [33] predict ferromagnetic order at the edges and antiferromagnetic inter-edge coupling at half filling.

The study of non-collinear magnetization has permitted to study the canted spin phases in graphene quantum Hall bars [28] as well as the existence of ingap topological fractional excitations at the domain walls of graphene zigzag ribbons [29].

\section{Proximity terms}

The effective Hamiltonian for electrons in graphene can be modified due to the interaction with the substrate. The most frequently considered types of proximity terms are a sublattice symmetry-breaking on-site potential, that opens up a gap [32, 34], a ferromagnetic spin proximity effect, that splits the bands [35-38] and a superconducting proximity effect, that adds a pairing term to the Hamiltonian, and opens up a superconducting gap to graphene whenever the Fermi energy lies on a band.

The on-site potential can be written down as:

$$
H_{J}=\sum_{i} W(i) c_{i \sigma}^{\dagger} c_{i \sigma}
$$

Whenever $W(i)$ is different for $A$ and $B$ sublattice, this term can open up a gap in graphene. When the sign of this gap is modulated across graphene, kink states can emerge [39].

The spin proximity effect can be written down as:

$$
H_{J}=\frac{1}{2} \sum_{i} \vec{J}(i) \cdot \vec{\sigma}_{\sigma, \sigma^{\prime}} c_{i \sigma}^{\dagger} c_{i \sigma^{\prime}}
$$

where $\vec{J}(i)$ is the exchange field that is proportional to the magnetization field of the proximity layer. In the simplest scenario, this is taken as a collinear and constant field, so that the spin proximity effect leads to a spin splitting of the bands that, in conjunction with Rashba spin orbit coupling, can induce a quantized anomalous Hall phase[35]. Spin proximity with non-collinear or even non-coplanar substrates, such as skyrmions, can also result in a quantized anomalous Hall phase, without the need of spin orbit coupling[40]. The typical magnitude for the exchange splitting, as obtained from DFT calculations [36-38], is in the range of a few tens of $\mathrm{meV}$ at most. Experimentally, a report of splitting induced by spin proximity effect observed in graphene is much smaller than that, in the range of a fraction of $\mathrm{meV}$ [41].

The superconducting proximity effect is introduced as an effective conventional s-wave pairing term:

$$
H_{S C}=\Delta \sum_{i}\left[c_{i, \uparrow} c_{i, \downarrow}+c_{i, \downarrow}^{\dagger} c_{i, \uparrow}^{\dagger}\right]
$$


This term has to be treated using the so called Bogoliubov de Gennes (BdG) Hamiltonian[42].

\section{EMERGENT PHASES AND ZERO MODES}

\section{Single particle theory of Zero modes}

We consider graphene ribbons not too far from their charge neutrality point. Therefore, their Fermi energy lies close to the Dirac point. Because of quantum confinement, extended states of graphene ribbons are gapped. This leads to semiconducting or insulating ribbons that are not expected to host nontrivial electronic phases. The way out of this situation comes from the existence of zero modes. In pristine GNR, zero modes arise in the following instances:

1. At sufficiently long zigzag edges[17]. As we discuss below, there is one zero mode for every three carbon atoms in a zigzag edge.

2. At interfaces between gapped armchair ribbons with different symmetry protected topological indexes $Z_{2}$, defined below, as proposed by $\mathrm{Cao}$ et al. [12]

In addition, zero modes also appear when graphene is functionalized with atomic hydrogen [43-46] or any other $\mathrm{sp}^{3}$ functionalization 47].

There are two complementary ways to understand the emergence of these zero modes. The first way invokes the bipartite character of the honeycomb lattice and the emergence of at least $N_{A}-N_{B}$ zero modes [44, 48], where $N_{A}$ and $N_{B}$ are number of sites in the two sublattices that form a structure. In addition, the theorem permits to anticipate the sublattice polarized nature of the zero modes. This first method permits to predict the emergence of zero modes in $\mathrm{sp}^{3}$ functionalized graphene[44]. There, the $p_{z}$ orbital forms a strong covalent bond with an orbital of the functionalizing species, such as the $1 s$ orbital of atomic hydrogen. This takes away both 1 electron and 1 orbital from the $p_{z}$ array. This can be effectively modeled as a tight-binding model with a missing site [44, 49]. The sublattice imbalance argument can also be applied right away to the interface states between armchair ribbons, [13] shown in figure 3 . In the case of graphene zigzag edges, it can be invoked, although in a less rigorous manner. Locally, zigzag edges have sublattice imbalance, but globally, the structures have $N_{A}=N_{B}$.

The existence of zero modes in some GNR can also be related to topological arguments. The interface between two media described with different topological indexes, $N_{1}$ and $N_{2}$, is expected to host at least
$N_{1}-N_{2}$ zero modes. In a $1 \mathrm{D}$ crystal with mirror and inversion symmetry, we define the Zak phase of a band $n$ as [12, 50]:

$$
\gamma_{n}=i\left(\frac{2 \pi}{d}\right) \int_{-\pi / d}^{\pi / d} d k\left\langle u_{n k} \mid \frac{\partial u_{n k}}{\partial k}\right\rangle
$$

where $d$ is the unit cell size and $u_{n k}$ is the periodic part of the Bloch wave function for band $n$. In a symmetry protected 1D crystal, the Zak phase is quantized as 0 or $\pi$ modulo $2 \pi$. This permits to define a topological index:

$$
(-1)^{Z_{2}}=e^{i \sum_{n} \gamma_{n}}
$$

From the bulk-boundary correspondence, symmetric junctions of armchair ribbon with different Z2 numbers are expected to host localized zero modes at the interfaces. This has been confirmed both with DFT [12] and tight-binding calculations[13]. These junctions happen to have $\left|N_{A}-N_{B}\right|=1$, so that the interface zero mode can be understood using the theorem for bipartite lattice. Using similar arguments[50], the existence of edge modes in 1D zigzag edges has been related to the Zak phase for the family of $1 \mathrm{D}$ states defined in a cut of the $2 \mathrm{D}$ Brillouin zone.

\section{Infinite ribbons}

We begin our discussion of specific systems with the case of one dimensional graphene ribbon with zigzag edges. As shown in figure 2(a), the energy bands feature two flat bands at $E=0$. These two bands of zero modes occupy exactly one third of the Brillouin zone. Given that the unit cell of the $\mathrm{ZZ}$ GNR has exactly one carbon site per edge, this implies that the ratio of zero modes per carbon edge atom is $1 / 3$. The wave function of the edge modes is sublattice polarized, and its amplitude quickly decays as we move inwards in the GNR. Other than these zero modes, the rest of the bands are gapped, reflecting the confinement of the Dirac particles in the section of the ribbon.

The flat bands at $E=0$ give rise to a very large density of states at that energy. Given that $E=0$ is the Fermi energy for half filling, interactions are expected to have a strong impact in this system. This was found out more before the turn of the century, using a mean field approximation for the Hubbard model in this system[23] and subsequent work, using both the Hubbard model[25,-27] and DFT calculations. In all instances, the predictions of these symmetry breaking methods are: 
(a)

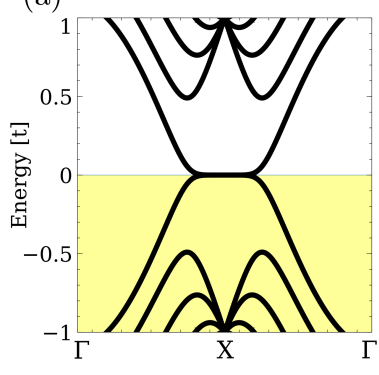

(b)

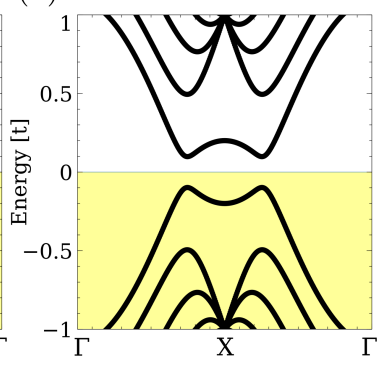

(c)

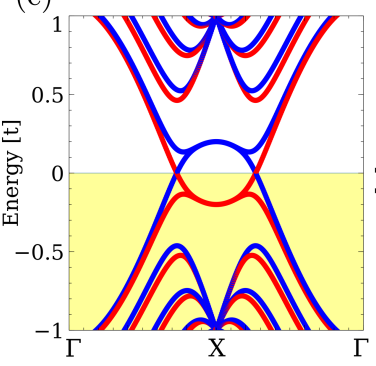

(d)

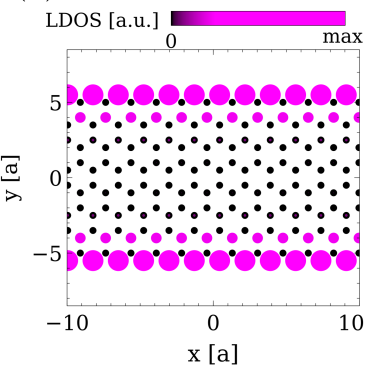

FIG. 2. (a) Band structure of a zigzag nanoribbon with a 16 atoms width. Band structure with local exchange fields in the upper and lower edges, aligned antiferromagnetically (b) and ferromagnetically (c). (d) Spatial distribution of the flat band of (a), that become magnetized in panels (b,c).

- The zigzag edges are ferrromagnetic, with magnetic moments in the range of $0.15 \mu_{B}$ per carbon atom

- The inter-edge interaction is antiferromagnetic and decays rapidly as a function of the width

- The energy bands, show a dispersion of the edge states, driven by the interactions [25]. In the case of parallel (antiparallel) alignment of the edge magnetizations, the $\mathrm{ZZ}$ is a conductor (insulator). This finding prompted proposals for using graphene ribbons as ideal spin valves [26, 51].

The qualitative effect of interactions can be captured by adding local exchange fields at the upper and lower zigzag edge, giving rise to results comparable to the full selfconsistent calculation (Fig. 2 $2 \mathrm{~b}, \mathrm{c})$ ).

In general, mean-field calculations for any nanographene with zigzag edges predicts the existence of magnetic moments localized at the edges with ferromagnetic correlations between edges that belong to the same sublattice, and antiferromagnetic correlations between edges that belong to opposite sublattices[24]. In the case of infinite 1D ribbons, these calculations have an obvious problem: they predict infinitely long range order along the edge, breaking a continuous symmetry in one dimension. This is incompatible with well established theorems. In one dimension, quantum fluctuations are known to destroy this type of long range order. Therefore, we need to carry out a treatment that models this system without this drawback. Before doing that, a possible way out would be to include the terms in the Hamiltonian that break the SU(2) spin symmetry, given that $1 \mathrm{D}$ order is possible at $T=0$ in Ising chains, for instance. It has been shown[27] that intrinsic spin orbit coupling favors in-plane edge mag- netization. As a result, the group of symmetry is reduced, but is still a continuous $\mathrm{O}(2)$ symmetry, for which no long range order can exist in 1D. In addition, the value of the magnetic anisotropy scales with the square of the intrinsic spin-orbit coupling term in the Kane-Mele Hamiltonian, which is in the range of a few tens of $\mu \mathrm{eV}$ in graphene[21]. Therefore, the magnetic anisotropy driven by the intrinsic spin orbit coupling in graphene is negligible.

The effect of spin wave fluctuations was considered by Yazyev and Katnelson [52]. They computed the spin correlation functions along the edges using a spin ladder model of an infinite ribbon and found a power law decay, with temperature dependence spin correlation length. A Quantum Monte Carlo description for the same a spin ladder model for zGNR was also carried out [53]. Beyond mean field explorations of edge ferromagnetism in zGNR has also been addressed out with fermionic models including long range Coulomb interactions using both exact diagonalizations in a restricted active space in the reciprocal state [54] as well as Quantum Monte Carlos simulation [55]. Both methods confirm intra-edge ferromagnetic correlations. In any event, it is apparent that a rigorous quantum theory for the edge magnetism has to go beyond broken symmetry solutions in order to include a proper treatment of quantum fluctuations.

\section{Finite ribbons}

We now consider two different finite size ribbons (see figure 3). Both of them have two weakly hybridized zero modes. The first one is a ribbon with two long armchair edges and two short zigzag edges, shown in figure 3 that hosts just one edge state each. The second structure combines armchair ribbons with 

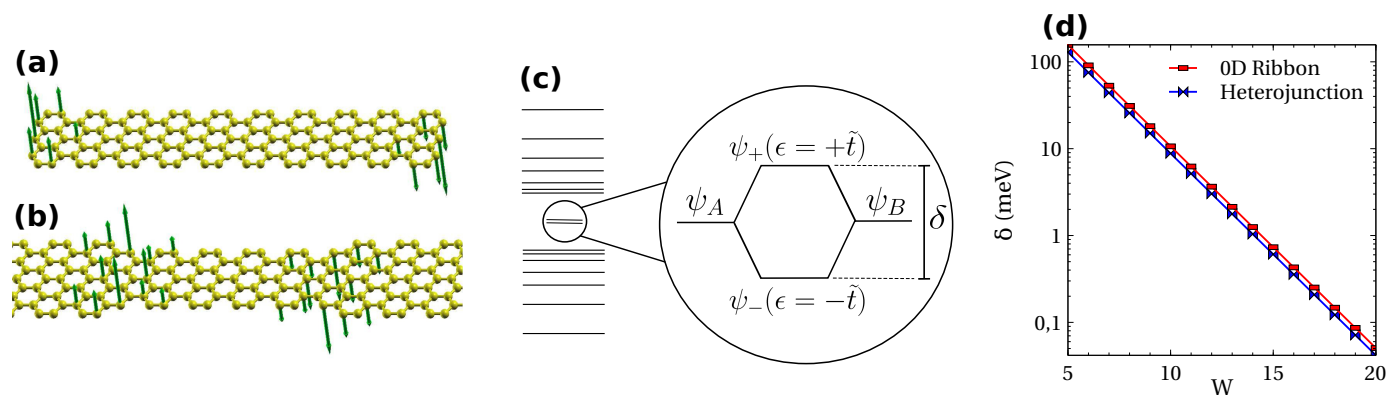

FIG. 3. Left: GNR with short zigzag edges (top) and aGNR with two widths. Both structures have in-gap states that host local moments when Coulomb interactions are included. The vector maps reflect the magnetization obtained in a mean field calculation with the Hubbard model. Right: Scheme of the single-particle $U=0$ energy spectrum showing two almost degenerate in-gap states. Their wave functions are the symmetric and antisymmetric combinations of the sublattice polarized zero modes located at the edges/interfaces. The exponential dependence of the hybridization, as measured by the in-gap splitting, as a function of $W$, the length scale that controls the size of the GNR.

different width and mirror symmetry, such that the interface hosts zero modes. For these structure, we take periodic boundary conditions so that there are no free zigzag edges. In both cases, the structures have two zero modes inside a quite large gap. At half filling, the two edge modes host one electron. We can treat these systems, including interactions, by considering configurations where the valence state are doubly occupied and the conduction states are empty. Therefore, we have a problem of 2 electrons in two sites, that can be solved analytically[13]. In both structures, we can change the dimensions of the system, and thereby $W$, defined as the that the distance between either the edge or the interface that controls the hybridization of the zero modes.

The non-interacting spectrum. A scheme of the single-particle spectrum characteristic of these gapped OD GNR with two in-gap states is shown in figure 3 (b). The energies and wave-functions of the in-gap states are denoted by $\varepsilon_{ \pm}$and $\psi_{ \pm}$respectively. It is always possible[13] to write down the wave function of a couple of conjugate states, with single-particle energy $E$ and $-E$, in terms of the same sublattice polarized states $\psi_{A}$ and $\psi_{B}$. Therefore, we write

$$
\begin{aligned}
& \psi_{A}(i) \equiv \frac{1}{\sqrt{2}}\left(\psi_{+}(i)+\psi_{-}(i)\right) \\
& \psi_{B}(i) \equiv \frac{1}{\sqrt{2}}\left(\psi_{+}(i)-\psi_{-}(i)\right)
\end{aligned}
$$

In the case of the in-gap states, the resulting $\psi_{A}$ and $\psi_{B}$ are spatially separated. This accounts, in part for the fact that the energy splitting of the zero modes, defined as:

$$
\delta=2\left\langle\psi_{A}\left|\mathscr{H}_{0}\right| \psi_{B}\right\rangle \equiv 2 \tilde{t}
$$

is small. In figure 3 (c) we plot $\delta$ for both the rectangular and the heterojunction nanographene, both with two in-gap states. It is apparent and well known[17] that this quantity decays exponentially with $W$. In the limit where $W$ is very large (see figure 3 (c)), $\delta$ vanishes, and the energy of the in-gap states goes to $E=0$, showing that these sublattice polarized states are zero modes.[17]

$$
U \neq 0
$$

We now consider the effect of interactions and show how it leads to the formation of local moments at the location of the hybridized zero modes.[13, [56]. The two energy scales that govern the low energy behavior for the two electrons in the two in-gap levels are $\delta$ and the energy overhead associated to doubly occupy the sublattice polarized states:

$$
\tilde{U}=U \sum_{i}\left|\psi_{A}(i)\right|^{4}=U \sum_{i}\left|\psi_{B}(i)\right|^{4}=U \eta
$$

The addition energy is thus given by the product of the atomic Hubbard $U$ and $\eta$, is the inverse participation ratio of the zero mode states. Our numerical calculations for the two structures of figure (14) yield $\eta=0.11$ for the zigzag edge states and $\eta=0.035$ for the interface states. We found that, as opposed to the case of $\delta, \eta$ has a very weak dependence of $W$. We take $U=|t|=2.7 \mathrm{eV}$. Therefore, the effective Hubbard interaction $\tilde{U}$ is in the range of 270 and $94 \mathrm{meV}$, for edge and interface states, respectively. 

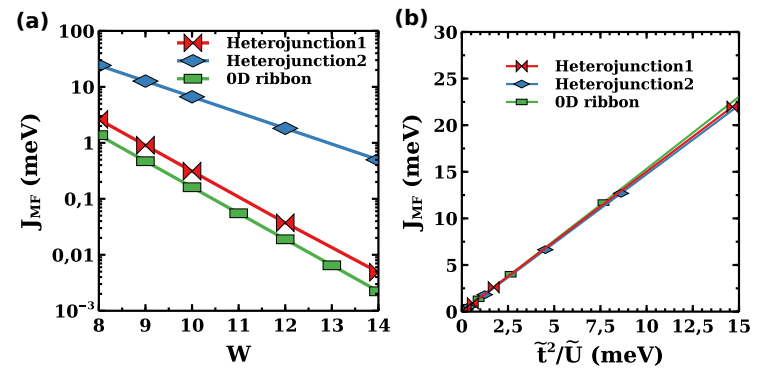

FIG. 4. Exchange couplings for OD ribbons and heterojunctions as a function of lateral dimension $W$ and as function of $\frac{\tilde{t}^{2}}{\tilde{U}}$. In the case of the heterojunctions, we compute independently the coupling mediated by either the wide or the narrow GNR, by adequate choice of the unit cell dimensions

\section{Mean field Treatment}

We discuss qualitatively the results of a mean field approximation for the Hubbard model for the two nanographenes of figure(14). The results are obtained using the collinear mean field treatment ( eqs. 677). For all structures for which $\tilde{U} \gg \delta$ we found broken symmetry solutions with a finite local magnetization, $M(i)=\left\langle S_{z}(i)\right\rangle$ that are mostly located in the region where either $\psi_{A}$ or $\psi_{B}$ are non-zero. The results of the magnetization field are shown in the left panels of figure (14). The net magnetization per zero mode is close to $S=1 / 2$.

Using the mean field approach, we can study the exchange energy as the difference between FM and $\mathrm{AF}$ solutions $J_{M F}=E_{F M}-E_{A F}$ as a function of $W$, for both types of structures. The FM and AF solutions are obtained by suitably forcing the selfconsistent iterative procedure to solve the mean field Hamiltonian. We show in figure 4 that $J_{M F}$ can be as large as $40 \mathrm{meV}$ can be made small by increasing the distance $W$ between the zero modes. Importantly, as we show in figure 4 (b), we find that, both for ribbons and heterojunctions, exchange energy scales as

$$
J_{M F} \propto \frac{\tilde{t}^{2}}{\tilde{U}}
$$

This scaling provides a strong indication that the mechanism of antiferromagnetic interaction is kinetic exchange [57, 58], that arises naturally for half-filled Hubbard dimers. The fact that local moments are hosted mostly by the in-gap states permits to build a restricted model where only the in-gap states are considered. This is the topic of the next paragraph.

\section{Effective Hubbard dimer}

In order to go beyond the mean field picture and to be able to describe local moments in these nanographenes with a full quantum theory without breaking symmetry, we restrict the Hilbert space to the configurations of 2 electrons in the two zero modes. To do so, we represent the Hubbard interaction in the one body basis defined by the states $\psi_{A}$ and $\psi_{B}$. The Hamiltonian so obtained is a two site Hubbard model with renormalized hopping and onsite energy [13, 56]:

$$
\mathscr{H}_{\mathrm{eff}}=\tilde{t} \sum_{\sigma}\left(a_{\sigma}^{\dagger} b_{\sigma}+b_{\sigma}^{\dagger} a_{\sigma}\right)+\tilde{U}\left(n_{A \uparrow} n_{A \downarrow}+n_{B \uparrow} n_{B \downarrow}\right)
$$

where $a_{\sigma}^{\dagger}=\sum_{i} \psi_{A}(i) c_{i \sigma}^{\dagger}$ and $b_{\sigma}^{\dagger}=\sum_{i} \psi_{B}(i) c_{i \sigma}^{\dagger}$ are the operators that create an electron in the zero modes $\psi_{A}$ and $\psi_{B}$ with spin $\sigma$, respectively. In turn, $n_{A, \sigma}=$ $a_{\sigma}^{\dagger} a_{\sigma}$ is the number operator for the $\psi_{A}$ state with $\operatorname{spin} \sigma$.

Hamiltonian (18) is a two-site Hubbard model, where the sites correspond to the zero mode states $\psi_{A, B}$, shown in figure $3(\mathrm{~b}, \mathrm{c}, \mathrm{d}, \mathrm{e})$. For the relevant case of 2 electrons, the dimension of the Hilbert space is 6 and the ground state is always a singlet, as inferred both from analytical solution [59] or by a straight-forward numerical diagonalization[13].

The exact solution permits to set the language to discuss the emergence of local moments in these structures. For this matter, we can write the wave function of the ground state as:

$$
\left|\Psi_{G S}\right\rangle=c_{2}(|2,0\rangle+|0,2\rangle)+c_{S}(|\uparrow, \downarrow\rangle-|\downarrow, \uparrow\rangle)
$$

where $|2,0\rangle$ describes a state with 2 electrons in one site of the Hubbard dimer, and none on the other, whereas $\left|\sigma_{1}, \sigma_{2}\right\rangle$ describes states with the one electron per site, with spins $\sigma_{1}, \sigma_{2}$. In figure we show hot, for $U=0$, we have $c_{2}=c_{S}=\frac{1}{2}$ so that double occupancy is as likely as individual occupancy. As $U$ is ramped up, the $c_{2}$ coefficient is depleted and the $c_{S}$ coefficient is enhanced, as shown in figure .

In order to characterize the magnetic behavior of the dimer, we define the spin operators:

$$
\begin{aligned}
& S_{z}(a) \equiv \frac{1}{2}\left(a_{\uparrow}^{\dagger} a_{\uparrow}-a_{\downarrow}^{\dagger} a_{\downarrow}\right) \\
& S_{z}(b) \equiv \frac{1}{2}\left(b_{\uparrow}^{\dagger} b_{\uparrow}-b_{\downarrow}^{\dagger} b_{\downarrow}\right)
\end{aligned}
$$

We can see right away that their expectation values are zero for the ground state, in contrast with the broken symmetry solutions of the mean field theory. We thus look up at the next moment, the spin correlation function. In particular, we can obtain the following result for the spin correlator for the ground state 
(a)

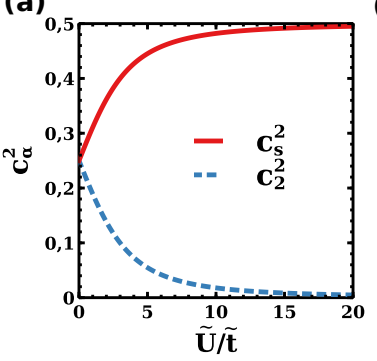

(b)

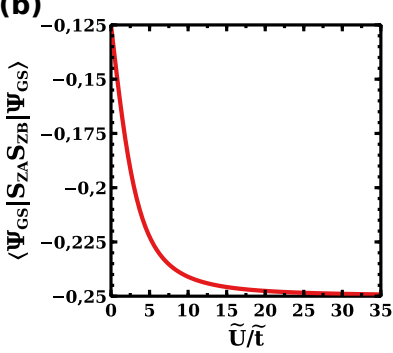

(a)

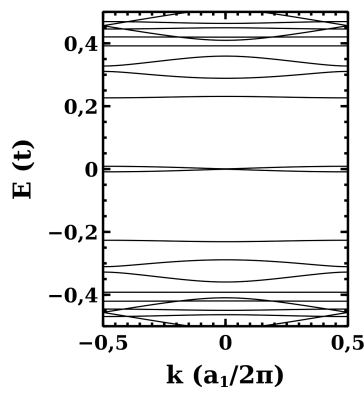

(b)

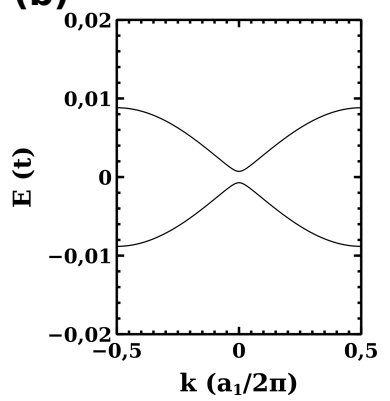

FIG. 5. Left: Evolution of the double occupancy and single occupancy weights in the ground state of the Hubbard dimer at half filling as a function of $U / t$. Right: evolution of the spin correlation function.

$\left\langle\Psi_{G S}\left|S_{z}(a) S_{z}(b)\right| \Psi_{G S}\right\rangle=-\frac{c_{S}^{2}}{2}$. Thus, for $U=0$ there is some spin correlation $(-1 / 8)$. As $U$ is ramped up, the correlation tends to $-1 / 4$, the value expected for $c_{S}=1 / \sqrt{2}$ and $c_{2}=0$. In that limit, wave function is identical to the spin singlet of the antiferromagnetic Heisenberg dimer.

In the strong coupling limit, $\tilde{U}>>\tilde{t}$, it is well known[57, 58] that the four lowest levels in the model of equation 18 can be mapped into the Heisenberg Hamiltonian:

$$
\mathscr{H}_{\mathrm{Heis}}=J_{H} \vec{S}_{A} \cdot \vec{S}_{B}
$$

where $J_{H} \simeq \frac{4 \tilde{t}^{2}}{\tilde{U}}$. The Hamiltonian of equation 21 , has a ground state singlet $(S=0)$ as well as an excited state triplet with $S=1$, separated in energy by $\Delta=$ $E(S=1)-E(S=0)=J_{H}$.

So, the picture that emerges from this model is the following: both structures considered here have two in-gap states each. These in-gap states have a splitting $\delta$ that arises from the hybridization of 2 sublattice polarized zero modes. The system is thus modeled with a Hubbard dimer. At half filling, the Hubbard dimer can be effectively mapped into a spin model, when the energy cost of double occupancy of these zero modes, given by equation $[16$ is much larger than the hybridization splitting.

The next question we address is how to up-scale these Hubbard dimers to obtain larger structures. In other words, how to couple more dimers together. There are at least two ways in which can do this. If we increase the width of the square shape graphene ribbon, making the zigzag edges wider, we shall increase the number of zero modes. Eventually, this leads to the case of $1 \mathrm{D}$ channels with ferromagnetic interactions, discussed in the previous section. The other way, is discussed in the next section.

FIG. 6. (a) Bands of a GNR made of two armchair sections with different width $\left(W_{t}=12, W_{n}=8\right.$. (b) Zoom showing the in-gap narrow bands, that arise from the interface zero modes, featuring a dimerization splitting at $k=0$, coming from the different intra and intercell hybridization of the interface zero modes.

\section{DIMERIZED SPIN CHAIN}

We consider a one dimensional ribbon with armchair edges and alternating section, whose unit cell contains two interface (quasi)-zero modes. The unit cell is described by two length scales, $W_{t}$ and $W_{n}$ that describe the width of the thicker and narrower AGNR. These two length scales control the effective hopping between zero modes. The zero modes thus lead to the formation two bands, inside the gap of the AGNR, shown in figure 6. These in-gap bands are effectively described by the $t, t^{\prime}$ model Hamiltonian. Their bandwidtt, governed the effective hybridization of the interface states, can be tuned by changing $W$, and can easily much smaller than the band-gap of these ribbons. In figure 6 we show a bandwidth of $0.02 \mathrm{t}$ inside a gap of $0.5 t$.

For $t \neq t^{\prime}$ the two bands have a gap at $k=\pi / L$, where $L$ is the length of the unit cell. The gap closes at $t=t^{\prime}$. This point separates two insulating phases $t>t^{\prime}$ and $t<t^{\prime}$ that are topologically distinct and have a different Zak phase[50]. As a result, only one of them has zero modes the edges. This happens when the last dimer is affected by the smallest of $t$ and $t^{\prime}$

We now consider the effect of interactions in the strong coupling limit $\tilde{U} \gg \tilde{t}, \tilde{t^{\prime}}$. Using the results of the previous section, and in line with previous work for other GNR structures [53, 60], we consider a spin model[61]

$$
\mathscr{H}_{\text {chain }}=\sum_{n=1, N}(J+\delta) \vec{S}_{2 n-1} \cdot \vec{S}_{2 n}+(J-\delta) \vec{S}_{2 n} \cdot \vec{S}_{2 n+1}
$$

The different exchanges are related to the different 
(a)
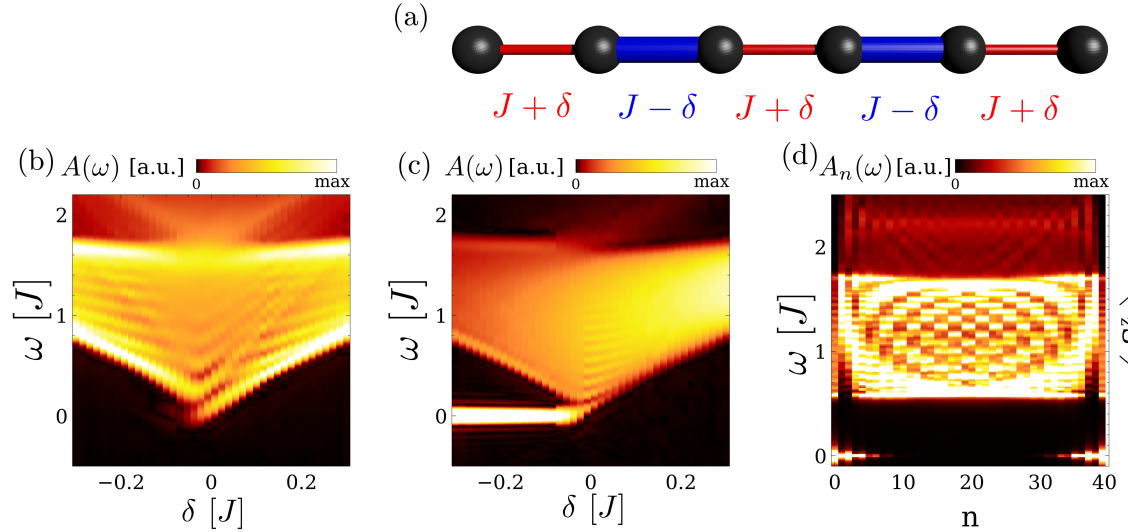

(e)

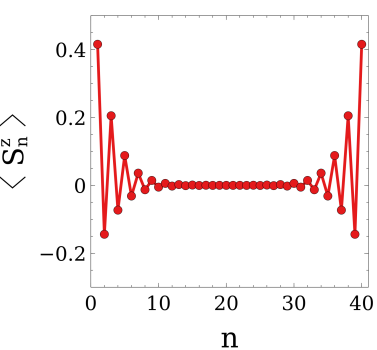

FIG. 7. (a) Sketch of a dimerized Heisenberg $S=1 / 2$ spin chain, having two exchange constants with different strength. Bulk (b) and edge (c) spectral function of the Heisenberg model, showing a gap for $\delta \neq 0$ in the bulk (b), while zero modes for $\delta<0$ on the edge. Panel (d) shows the spectral function in every site, showing the edge localization of the zero modes. Panel (e) shows the local magnetization under a small external field $B_{z}=0.01 \mathrm{~J}$, showing the localization of the emergent edge modes.

hoppings where we can write up:

$$
J_{n}=4 \frac{\tilde{t}_{n}^{2}}{\tilde{U}} ; \quad J_{t}=4 \frac{\tilde{t}_{t}^{2}}{\tilde{U}}
$$

We now have $J_{t}-J_{n}=2 \delta$ and $J_{t}+J_{n}=2 J$.

The previous model can be easily solved $(22)$ for a chain of $N=40$ sites ( 20 dimers) using density matrix renormalization group [62-68] implemented in the matrix product formalism[69-71] (DMRG). In particular, this method permits to obtain the expectation values and correlation functions of spin operators the ground state in a computationally efficient manner. The matrix product formalism also allows to access dynamical quantities of many body systems, 72 74]. Specifically, in the following we discuss the dynamical structure factor defined as

$$
A_{n}(\omega)=\left\langle\Psi_{G S}\left|S_{n}^{z} \delta\left(\omega-\mathscr{H}+E_{G S}\right) S_{n}^{z}\right| \Psi_{G S}\right\rangle
$$

The local dynamical structure factor can be qualitatively understood as the quantity giving access to the local density of states of the many body spin excitations. Using this method, we find that, in bulk, there is a gap for $\delta \neq 0$ (see Figure 7)(b). The edge structure factor shows gapless states for $\delta<0$ (see figure 7)(c). In figure 7(d) we show the map of the spectral function as a function of position and energy, for a a fixed value of $\delta=-0.2 J$. It is apparent that both edges host zero modes. This phenomenology is similar to the one of the SSH model, and therefore this system can be intuitively understood as a many-body version of a symmetry-protected topological state.[75]
The edge topological excitations can be accessed by means of a weak external field. First, it is important to note that, for sufficiently large chains, the dimerized Heisenberg model has a ground state that is four fold degenerate when $\delta<0$, consisting on a singlet and a triplet state, stemming from the dangling edge excitations. Upon an introduction of a weak external field, the triplet state with $S_{z}=+1$ becomes the ground state, giving rise to a finite magnetization in the edges. In figure 7( e) we show the local expectation value of $S_{z}$ for the $N=40$ chain with a small external field of $B_{z}=0.01 \mathrm{~J}$.

\section{MAGNETIC RIBBONS COMPETING A SUPERCONDUCTING PROXIMITY EFFECT}

In this section we discuss a situation where graphene ribbons that host local moments, driven by the exchange interactions discussed above, are in addition exposed to superconducting proximity effect coming from the substrate. This system permits the study of the competition between magnetism and superconductivity.

We consider first the energy spectrum of the ribbon without magnetic order and with a proximity pairing $\Delta$ in the Bogoliubov -de Gennes (BdG) Hamiltonian [42, 76, 77]. As we show in figure 8 (a), superconducting proximity opens up a gap $\Delta$ at the Fermi energy. Time reversal symmetric perturbations can modify the spectrum, but the energy levels can not be inside the gap.

Things are different when we consider the effect of 


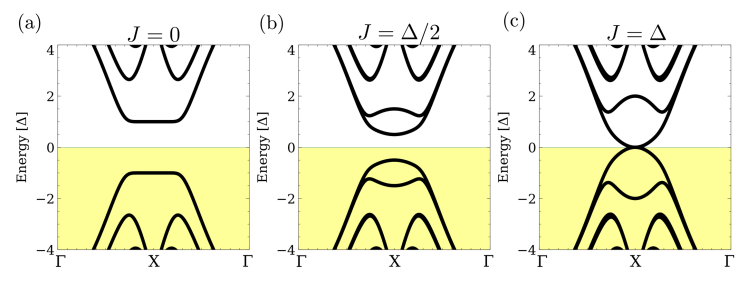

FIG. 8. (a,b,c) Bogoliubov-de-Gennes spectra for a zigzag ribbon with superconducting proximity effect, for different values of the edge exchange $J$, showing the closing of the gap as $J$ increases. The width of the ribbon in 16 atoms, the edges are in the AF configuration and we took $\Delta=0.2 t$

the magnetic order at the edges. We follow our previous work [77] and we model the exchange by adding a spin and position dependent on-site potential:

$$
V_{\text {exch }}=\sum_{i \in \text { edge }} \frac{J(i)}{2}\left(n_{i \uparrow}-n_{i \downarrow}\right)
$$

where $J(i)=0$ everywhere except at the top edge, for which $J(i)=J$ and the bottom edge, for which $J(i)= \pm J$. We thus consider two different relative orientations of the edge magnetization. The modification of the energy bands and density of states of the superconducting ribbon due to the exchange is shown in figures 8 and 9 , respectively. The most outstanding feature is the emergence of in-gap YuShiba-Rusinov (YSR) -[78-80] states. Interestingly, we find an energy dependence of the YSR states linear in $J$, in line with the one obtained for hydrogenated graphene [77], but different from the standard non-linear dependence of YSR states in normal metals.

The effect of the increasing exchange coupling can be also observed in the Bogoliubov-de-Gennes excitation spectra of the ribbon as shown in Fig. 8. First, in the absence of exchange field a superconducting gap opens up. As the exchange field increases, the gap starts closing until at critical value the system becomes gapless. This phenomenology is similar to the one found in single magnetic impurities, [81] where as $J$ is increased a single in-gap excitation approaches the charge neutrality point, giving rise to a parity switching point. In the case of graphene nanoribbons the behavior is more complex due to the existence of several YSR branches that give rise a to a continuum of in-gap excitations. [82, 86$]$
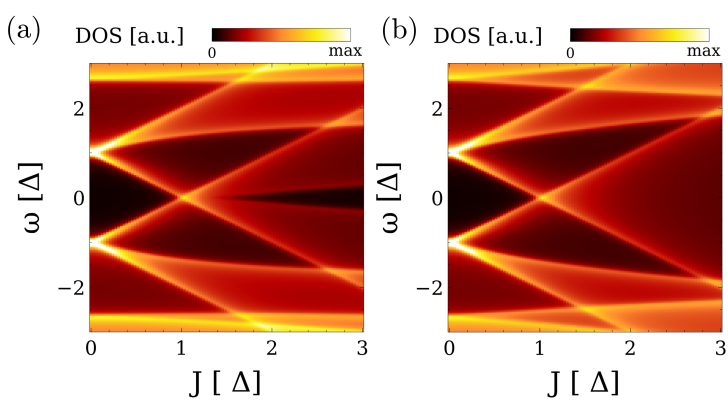

FIG. 9. Evolution of the DOS for $1 \mathrm{D} z \mathrm{z}$. $\mathrm{DN}$ with superconducting proximity effect as the spin-splitting $J$ induced by ferromagnetic edge order. Left: antiparallel inter-edge alignment. Right: parallel inter-edge alignment. In both instances in-gap states are induced as $J$ is ramped up. We took a width of 16 atoms and $\Delta=0.2 t$.

\section{EXPERIMENTAL PROBES}

The experimental study of the non-trivial electronic phases discussed in this chapter can be carried out with state of the art scanning tunnelling microscope (STM) spectroscopy. More specifically, the collective spin excitations of either the magnetically ordered phase, or those of the spin-liquid phase of the dimerized spin chain could be probed with inelastic electron tunneling spectroscopy (IETS) [7, 87, 88]. Spin excitations in the range of a few meV can be resolved, and the mapping of their intensity profile across the ribbons can help to discriminate from other inelastic excitations in the system, such as phonons.

Given that both the synthesis and the STM probing require to have the GNR on top of a conducting surface, there will be spin exchange interactions between the local moments at graphene and the conduction electrons at the surface, that we have ignored so far. These Kondo interactions can compete with the exchange interactions discussed so far in several ways. First, if sufficiently strong, Kondo effect could screen the local moments in graphene. Recent experiments in GNR studied with STM spectroscopy show Kondo peaks[7], that very likely imply the screening of the graphene local moment. Second, additional indirect exchange interactions, mediated by the substrate electrons, can compete, or perhaps enhance, the graphene mediated interactions. In any event, these Kondo interactions will affect the lineshapes of the inelastic electron tunneling spectra measured with STM [89] 


\section{CONCLUSIONS AND OUTLOOK}

We have explored the several examples of emergence of non-trivial quantum phases in graphene nanoribbons. The building blocks for these phases are zero modes that form narrow bands at the Dirac energy. The band-width of these bands depends on the aspect ratio of the structures, which provides thereby a control knob. We have focused mostly on the case of neutral GNR, that leads to half-full narrow bands that result in insulating structure with interesting spin physics but frozen charge dynamics. Departure from half-filling is expected to result in very interesting electronic properties. For instance, doping the 1D ferromagnetic edge is expected to result in domain walls that host fractionalized electrons [29]. Doping the dimerized spin chains might result in superconducting phases. Superconducting proximity effect can be another way to explore the interplay between spin and charge. We have discussed the emergence of in-gap Shiba bands in graphene ribbons. When non-collinear magnetic ground states and/or spin-orbit coupling are considered, the Shiba bands could give rise to topological superconductivity with Majorana end modes [82].

Acknowledgments J. F.-R. acknowledge financial support from FCT for the P2020PTDC/FIS-NAN/4662/2014, the P2020PTDC/FIS-NAN/3668/2014 and the UTAPEXPL/NTec/0046/2017 projects, as well as Generalitat Valenciana funding Prometeo2017/139 and MINECO Spain (Grant No. MAT2016-78625-C2). R.O.C. acknowledges "Generalitat Valenciana" and "Fondo Social Europeo" for a Ph.D. fellowship (ACIF/2018/198). J. L. L. acknowledges financial support from the ETH Fellowship program.

[1] S. Wang, L. Talirz, C. A. Pignedoli, X. Feng, K. Müllen, R. Fasel, and P. Ruffieux, Nature Communications 7, 11507 (2016).

[2] C. Tao, L. Jiao, O. V. Yazyev, Y.-C. Chen, J. Feng, X. Zhang, R. B. Capaz, J. M. Tour, A. Zettl, S. G. Louie, et al., Nature Physics 7, 616 (2011).

[3] P. Ruffieux, J. Cai, N. C. Plumb, L. Patthey, D. Prezzi, A. Ferretti, E. Molinari, X. Feng, K. Mllen, C. A. Pignedoli, et al., Acs Nano 6, 6930 (2012).

[4] P. Ruffieux, S. Wang, B. Yang, C. Sánchez-Sánchez, J. Liu, T. Dienel, L. Talirz, P. Shinde, C. A. Pignedoli, D. Passerone, et al., Nature 531, 489 (2016).

[5] P. H. Jacobse, A. Kimouche, T. Gebraad, M. M. Ervasti, J. M. Thijssen, P. Liljeroth, and I. Swart, Nature Communications 8 (2017), 10.1038/s41467-01700195-2
[6] F. Schulz, P. H. Jacobse, F. F. Canova, J. van der Lit, D. Z. Gao, A. van den Hoogenband, P. Han, R. J. K. Gebbink, M.-E. Moret, P. M. Joensuu, I. Swart, and P. Liljeroth, The Journal of Physical Chemistry C 121, 2896 (2017)

[7] J. Li, S. Sanz, M. Corso, D. J. Choi, D. Peña, T. Frederiksen, and J. I. Pascual, Nature communications 10, 200 (2019).

[8] L. Yan and P. Liljeroth, arXiv e-prints , arXiv:1905.03328 (2019), arXiv:1905.03328 [condmat.mtrl-sci]

[9] Y. Cao, V. Fatemi, A. Demir, S. Fang, S. L. Tomarken, J. Y. Luo, J. D. Sanchez-Yamagishi, K. Watanabe, T. Taniguchi, E. Kaxiras, et al., Nature 556, 80 (2018).

[10] Y. Cao, V. Fatemi, S. Fang, K. Watanabe, T. Taniguchi, E. Kaxiras, and P. Jarillo-Herrero, Nature 556, 43 (2018).

[11] O. Gröning, S. Wang, X. Yao, C. A. Pignedoli, G. B. Barin, C. Daniels, A. Cupo, V. Meunier, X. Feng, A. Narita, K. Müllen, P. Ruffieux, and R. Fasel, $\mathrm{Na}$ ture 560, 209 (2018).

[12] T. Cao, F. Zhao, and S. G. Louie, Physical review letters 119, 076401 (2017).

[13] R. Ortiz, N. A. García-Martínez, J. L. Lado, and J. Fernández-Rossier, Physical Review B 97, 195425 (2018).

[14] D. J. Rizzo, G. Veber, T. Cao, C. Bronner, T. Chen, F. Zhao, H. Rodriguez, S. G. Louie, M. F. Crommie, and F. R. Fischer, Nature 560, 204 (2018).

[15] A. C. Neto, F. Guinea, N. M. Peres, K. S. Novoselov, and A. K. Geim, Reviews of modern physics 81, 109 (2009).

[16] M. I. Katsnelson, Graphene: Carbon in Two Dimensions (Cambridge University Press, 2012).

[17] K. Nakada, M. Fujita, G. Dresselhaus, and M. S. Dresselhaus, Physical Review B 54, 17954 (1996).

[18] C. L. Kane and E. J. Mele, Phys. Rev. Lett. 95, 146802 (2005)

[19] J. Sichau, M. Prada, T. Anlauf, T. J. Lyon, B. Bosnjak, L. Tiemann, and R. H. Blick, Phys. Rev. Lett. 122, 046403 (2019)

[20] L. Kou, F. Hu, B. Yan, T. Wehling, C. Felser, T. Frauenheim, and C. Chen, Carbon 87, 418 (2015).

[21] H. Min, J. Hill, N. A. Sinitsyn, B. Sahu, L. Kleinman, and A. H. MacDonald, Physical Review B 74, 165310 (2006).

[22] T. O. Wehling, E. Şaşığlu, C. Friedrich, A. I. Lichtenstein, M. I. Katsnelson, and S. Blügel, Phys. Rev. Lett. 106, 236805 (2011)

[23] M. Fujita, K. Wakabayashi, K. Nakada, and K. Kusakabe, Journal of the Physical Society of Japan 65, 1920 (1996).

[24] J. Fernández-Rossier and J. J. Palacios, Phys. Rev. Lett. 99, 177204 (2007)

[25] J. Fernández-Rossier, Physical Review B 77, 075430 (2008).

[26] F. Muñoz Rojas, J. Fernández-Rossier, and J. J. Palacios, Phys. Rev. Lett. 102, 136810 (2009).

[27] J. L. Lado and J. Fernández-Rossier, Phys. Rev. Lett. 113, 027203 (2014)

[28] J. L. Lado and J. Fernández-Rossier, Phys. Rev. B 90, 165429 (2014) 
[29] M. López-Sancho and L. Brey, 2D Materials 5, 015026 (2017).

[30] J. Jung and A. H. MacDonald, Phys. Rev. B 79, $235433(2009)$

[31] O. V. Yazyev, Reports on Progress in Physics 73, 056501 (2010).

[32] D. Soriano and J. Fernández-Rossier, Phys. Rev. B 85, 195433 (2012)

[33] Y.-W. Son, M. L. Cohen, and S. G. Louie, Physical review letters 97, 216803 (2006).

[34] G. Giovannetti, P. A. Khomyakov, G. Brocks, P. J. Kelly, and J. van den Brink, Phys. Rev. B 76, 073103 (2007)

[35] Z. Qiao, S. A. Yang, W. Feng, W.-K. Tse, J. Ding, Y. Yao, J. Wang, and Q. Niu, Phys. Rev. B 82, 161414 (2010)

[36] H.-X. Yang, A. Hallal, D. Terrade, X. Waintal, S. Roche, and M. Chshiev, Physical review letters 110, 046603 (2013).

[37] A. Hallal, F. Ibrahim, H. Yang, S. Roche, and M. Chshiev, 2D Materials 4, 025074 (2017).

[38] C. Cardoso, D. Soriano, N. A. García-Martínez, and J. Fernández-Rossier, Phys. Rev. Lett. 121, 067701 (2018)

[39] R. Jackiw and C. Rebbi, Phys. Rev. D 13, 3398 (1976)

[40] J. L. Lado and J. Fernández-Rossier, Phys. Rev. B 92, 115433 (2015)

[41] J. C. Leutenantsmeyer, A. A. Kaverzin, M. Wojtaszek, and B. J. van Wees, 2D Materials 4, 014001 (2016).

[42] C. Beenakker, Physical review letters 97, 067007 (2006).

[43] O. V. Yazyev and L. Helm, Physical Review B 75, 125408 (2007).

[44] J. J. Palacios, J. Fernández-Rossier, and L. Brey, Phys. Rev. B 77, 195428 (2008)

[45] H. González-Herrero, J. M. Gómez-Rodríguez, P. Mallet, M. Moaied, J. Palacios, C. Salgado, M. M. Ugeda, J. Y. Veuillen, F. Yndurain, and I. Brihuega, Science 352, 437 (2016).

[46] N. A. García-Martínez, J. L. Lado, D. Jacob, and J. Fernández-Rossier, Phys. Rev. B 96, 024403 (2017)

[47] E. J. Santos, A. Ayuela, and D. Sánchez-Portal, New Journal of Physics 14, 043022 (2012).

[48] B. Sutherland, Phys. Rev. B 34, 5208 (1986)

[49] D. Soriano, F. Muñoz Rojas, J. Fernández-Rossier, and J. J. Palacios, Phys. Rev. B 81, 165409 (2010)

[50] P. Delplace, D. Ullmo, and G. Montambaux, Physical Review B 84, 195452 (2011).

[51] W. Y. Kim and K. S. Kim, Nature nanotechnology 3, 408 (2008).

[52] O. V. Yazyev and M. I. Katsnelson, Phys. Rev. Lett. 100, 047209 (2008)

[53] C. Koop and S. Wessel, Phys. Rev. B 96, 165114 (2017)

[54] Z. Shi and I. Affleck, Phys. Rev. B 95, 195420 (2017)

[55] M. Raczkowski and F. F. Assaad, Physical Review B 96, 115155 (2017).

[56] M. Golor, C. Koop, T. C. Lang, S. Wessel, and M. J. Schmidt, Physical review letters 111, 085504 (2013).
[57] P. W. Anderson, Phys. Rev. 115, 2 (1959)

[58] T. Moriya, Physical Review 120, 91 (1960).

[59] S. A. Jafari, Iranian Journal of Physics Research 8, 116 (2008)

[60] M. Golor, S. Wessel, and M. J. Schmidt, Phys. Rev. Lett. 112, 046601 (2014)

[61] R. Chitra, S. Pati, H. R. Krishnamurthy, D. Sen, and S. Ramasesha, Phys. Rev. B 52, 6581 (1995)

[62] S. R. White, Phys. Rev. Lett. 69, 2863 (1992)

[63] U. Schollwöck, Rev. Mod. Phys. 77, 259 (2005)

[64] E. Jeckelmann, Phys. Rev. B 66, 045114 (2002)

[65] U. Schollwöck, Annals of Physics 326, 96 (2011)

[66] E. M. Stoudenmire and S. R. White, Phys. Rev. Lett. 119, 046401 (2017)

[67] S. R. White, Phys. Rev. B 72, 180403 (2005)

[68] S. R. Manmana, E. M. Stoudenmire, K. R. A. Hazzard, A. M. Rey, and A. V. Gorshkov, Phys. Rev. B 87, 081106 (2013)

[69] F. Verstraete, V. Murg, and J. Cirac, Advances in Physics 57, 143 (2008)

[70] E. M. Stoudenmire and S. R. White, Phys. Rev. B 87, 155137 (2013)

[71] "Itensor, http://itensor.org/,"

[72] A. Weiße, G. Wellein, A. Alvermann, and H. Fehske, Rev. Mod. Phys. 78, 275 (2006)

[73] F. A. Wolf, I. P. McCulloch, O. Parcollet, and U. Schollwöck, Phys. Rev. B 90, 115124 (2014)

[74] J. L. Lado and O. Zilberberg, arXiv e-prints , arXiv:1906.07090 (2019), arXiv:1906.07090 [condmat.mes-hall]

[75] W. P. Su, J. R. Schrieffer, and A. J. Heeger, Phys. Rev. Lett. 42, 1698 (1979)

[76] P. San-Jose, J. L. Lado, R. Aguado, F. Guinea, and J. Fernández-Rossier, Phys. Rev. X 5, 041042 (2015)

[77] J. Lado and J. Fernández-Rossier, 2D Materials 3, 025001 (2016).

[78] L. Yu, Acta Phys. Sin 21, 75 (1965).

[79] H. Shiba, Progress of theoretical Physics 40, 435 (1968).

[80] A. Rusinov, Sov. Phys. JETP 29, 1101 (1969).

[81] A. V. Balatsky, I. Vekhter, and J.-X. Zhu, Rev. Mod. Phys. 78, 373 (2006)

[82] S. Nadj-Perge, I. K. Drozdov, J. Li, H. Chen, S. Jeon, J. Seo, A. H. MacDonald, B. A. Bernevig, and A. Yazdani, Science 346, 602 (2014)

[83] J. Röntynen and T. Ojanen, Phys. Rev. Lett. 114, 236803 (2015)

[84] G. C. Ménard, S. Guissart, C. Brun, S. Pons, V. S. Stolyarov, F. Debontridder, M. V. Leclerc, E. Janod, L. Cario, D. Roditchev, P. Simon, and T. Cren, Nature Physics 11, 1013 (2015)

[85] M. Ruby, Y. Peng, F. von Oppen, B. W. Heinrich, and K. J. Franke, Phys. Rev. Lett. 117, 186801 (2016)

[86] S. Kezilebieke, M. Dvorak, T. Ojanen, and P. Liljeroth, Nano Letters 18, 2311 (2018)

[87] C. F. Hirjibehedin, C. P. Lutz, and A. J. Heinrich, Science 312, 1021 (2006).

[88] J. Fernández-Rossier, Phys. Rev. Lett. 102, 256802 (2009)

[89] Y.-h. Zhang, S. Kahle, T. Herden, C. Stroh, M. Mayor, U. Schlickum, M. Ternes, P. Wahl, and K. Kern, Nature communications 4, 2110 (2013). 